Etienne Cavalier*, Pierre Lukas and Pierre Delanaye

\title{
Analytical evaluation of the Nittobo Medical tartrate resistant acid phosphatase isoform $5 b$ (TRACP-5b) EIA and comparison with IDS iSYS in different clinically defined populations
}

https://doi.org/10.1515/cclm-2021-1190

Received October 9, 2021; accepted December 6, 2021;

published online December 16, 2021

\section{Abstract}

Objectives: Tartrate-resistant acid phosphatase, isoform $5 b$ (TRACP-5b) is a bone resorption marker not influenced by renal function or food intake. TRACP-5b can be measured with Nittobo Medical enzymatic-immunoassay and IDS-iSYS automated immunoassay. We evaluated the Nittobo assay and established reference ranges for a Western-European population. We compared Nittobo and IDS results in different well-defined clinical populations. Methods: We established the limits of detection and quantification (LOD-LOQ), linearity, imprecision and the reference ranges in 119 males, 50 women ( $<45$ years) and 120 women ( $>60$ years) for TRACP-5b with the Nittobo assay. We compared both assays in 30 hemodialyzed (HD), and 40 stage 3-5 patients suffering from chronic kidney disease (CKD), 40 patients suffering from rheumatoid arthritis and osteoporosis and 80 post-menopausal women. We measured TRACP-5b, $\beta$-crosslaps ( $\beta$-CTX), bone alkaline phosphatase (B-ALP) and PTH in 20 hemodialyzed (HD) and 40 CKD patients.

Results: LOD and LOQ were 0.02 and $0.35 \mathrm{U} / \mathrm{L}$. CV ranged from 8.3 to $4.3 \%$ (2/5 samples presenting $\mathrm{CV}>$ desirable $\mathrm{CV})$. Method was linear up to of $11.3 \mathrm{U} / \mathrm{L}$. Upper and lower limits of normality were $0.8-7.6 \mathrm{U} / \mathrm{L}$ in men, $0.9-4.7 \mathrm{U} / \mathrm{L}$ in

\footnotetext{
*Corresponding author: Prof. Etienne Cavalier, Department of Clinical Chemistry, Service de Chimie Clinique, University of Liege, CHU de Liège, Domaine Universitaire du Sart-Tilman, 4000, Liège, Belgium, Phone: +3243667692 , Fax: +3243668823 ,

E-mail: etienne.cavalier@chuliege.be. https://orcid.org/0000-00030947-2226

Pierre Lukas, Department of Clinical Chemistry, University of Liege, $\mathrm{CHU}$ de Liège, Liège, Belgium

Pierre Delanaye, Department of Nephrology, Dialysis and

Hypertension, University of Liège, CHU de Liège, Liège, Belgium; and Department of Nephrology-Dialysis-Apheresis, Hôpital Universitaire Carémeau, Nîmes, France
}

women $<45$ and $0.9-7.1 \mathrm{U} / \mathrm{L}$ in women $>60$. The regression equation between the 2 methods was Nittobo $=1.13$ (95\% CI: 1.09-1.16) $\times$ iSYS - 0.4 (95\% CI: $-0.5 ;-0.3)$. TRACP-5b and b-ALP were in their respective reference ranges for most of CKD and HD patients. That was not the case for $\beta$-CTX, which increased with decreasing eGFR.

Conclusions: Nittobo TRACP-5b presents interesting analytical features and a good concordance with IDS iSYS. These methods could thus potentially be harmonized.

Keywords: analytical validation; bone resorption; bone turnover marker; harmonization; tartrate resistant acid phosphatase isoform $5 b$; TRACP-5b.

\section{Introduction}

Tartrate-resistant acid phosphatase (TRACP) is an enzyme produced by the ACP5 gene [1] which possesses a dimetal center comprised of two Fe ions in its active center [2]. TRACP is expressed by various cells from monocyte/macrophage lineage like osteoclasts, activated macrophages or dendritic cells [3]. Two isoforms of TRACP, namely $5 \mathrm{a}$ and $5 \mathrm{~b}$ are present in human serum [4]. The difference between the two isoforms is characterized by the post-translational modification of each derived cell. TRACP-5a concentrations are increased in inflammatory pathologies like rheumatoid arthritis whereas TRACP-5b is secreted by the osteoclasts as an active enzyme and reflects bone resorption and the number of active osteoclasts [5, 6]. After release in the circulation, TRACP-5b becomes inactive by losing its iron content and degraded into fragments that are cleared by the liver [7]. Less than $10 \%$ of the circulating TRACP-5b circulates as an intact enzymatically active form [8].

TRACP-5b is clinically used as a resorption marker and has some important advantages over C-terminal telopeptide of type I collagen ( $\beta$-CTX), the resorption marker recommended by the IOF-IFCC [9]. Indeed, TRACP-5b concentrations are neither influenced by chronic kidney disease (CKD) nor food intake [10]. TRACP-5b also presents a weak diurnal variation and a low intra-individual 
variability [11]. Finally, TRACP-5b is also very stable in serum [12]. According to these interesting features, TRACP-5b may be useful in different clinical situations such as osteoporosis (it is indeed the resorption marker recommended by the Japan Osteoporosis Society [13]) and in monitoring bone turnover in CKD, renal transplanted and hemodialyzed patients $[14,15]$ for whom it is considered as a suitable alternative for the monitoring of bone resorption [16]. In hemodialyzed patients, a TRACP-5b concentration $\leq 4.6 \mathrm{U} / \mathrm{L}$ was shown to be able to discriminate low from non-low bone turnover with a sensitivity of $89 \%$ and a specificity of $71 \%$ [17] and in stage 4 to 5D and kidney transplant recipients, TRACP-5b was the best biomarker to predict low bone turnover [18].

Ideally, assays should specifically measure the intact active form of TRAP- $5 \mathrm{~b}$, but not the "total"-5b, nor the $5 \mathrm{a}$ forms. Two assays with high specificity for the active TRACP- $5 \mathrm{~b}$ form have been developed and are available on the market. The first one is an Enzyme Immunoassay (EIA), developed by Nittobo Medical (Tokyo, Japan). The second one is the IDS TRACP-5b assay, available as an automated immunoassay on the iSYS platform or as an ELISA (Immunodiagnostic Systems, Boldon, UK). The Nittobo assay is largely used in Japan and the IDS assays are rather used in other parts of the world. However, the two methods have never been compared so far. In this study, we aimed at evaluating the Nittobo assay, and to verify if both Nittobo and IDS iSYS assays provide harmonized results.

\section{Materials and methods}

\section{Assays}

The Nittobo assay is a fragment absorbed immunocaptured enzymatic assay which uses two monoclonal antibodies (anti-active TRACP-5b and anti-inactive TRACP-5b antibodies) which enables highly specific TRACP-5b measurement without cross-reactivity with TRACP-5a. The Nittobo assay uses 2-chloro-4-nitrophenyl phosphate (CNPP) as substrate that is highly specific for TRACP-5b. The assay ranges from 0.1 to 15.0 U/L. The limit of detection (LoD) have been established at $0.1 \mathrm{U} / \mathrm{L}$ by the manufacturer. In a Japanese population, the expected concentrations are of 1.7-5.9 U/L in men, 1.2 to 4.2 in young adult mean (YAM) value in women [19]. The DYNEX DS2 ${ }^{\circledR}$ system (Chantilly, VA) was used to automatically process the EIA.

The IDS iSYS TRAcP 5b (BoneTRAP ${ }^{\circledR}$ ) assay is an automated immunoassay using magnetic beads to capture both TRACP- 5 forms by a biotinylated monoclonal antibody. Specificity for TRACP-5b is obtained by measuring enzyme activity using the substrate at the optimal $\mathrm{pH}$, specifically for type 5b. The IDS assay uses p-nitrophenylphosphate (pNPP) as substrate. The assay ranges from 0.9 to 14.0 U/L. The limit of detection (LoD) and the limit of quantification
(LoQ) have been established at $\leq 0.6 \mathrm{U} / \mathrm{L}$ and $\leq 0.9 \mathrm{U} / \mathrm{L}$ by the manufacturer, respectively. The total imprecision of the assay ranges from $13.6 \%$ at a value of $1.7 \mathrm{U} / \mathrm{L}$ to $5.0 \%$ at a value of $12.0 \mathrm{U} / \mathrm{L}$. Finally, the expected concentrations are of $1.4-6.1 \mathrm{U} / \mathrm{L}$ in men, 1.2 to 4.8 in premenopause women and 1.1-6.9 U/L in menopause women.

\section{Analytical validation protocol}

(1) The LoD and LoQ were assessed according to the CLSI EP 17 guideline. The LoD was established by running a blank sample and the lower calibrator in 5-plicates for 5 days and corresponded to the mean of the blank +2 standard deviations. The LoQ was established by measuring seven samples presenting low concentrations of TRACP-5b in 5-plicates on five consecutive days and was defined as the extrapolation of the value giving a coefficient of variation (CV) of $20 \%$.

(2) Intra- and inter-day imprecision was calculated by ANOVA on five native samples measured on 5-plicates on five consecutive days according to the CLSI EP 5A2 guideline. The target for desirable imprecision was defined as $50 \%$ of the intra-individual coefficient of variation $\left(\mathrm{CV}_{\mathrm{I}}\right)$ of TRACP-5b available on the EFLM Biological Variation Database (https://biologicalvariation.eu/) [20]. According to this database, the $\mathrm{CV}_{\mathrm{I}}$ of TRACP- $5 \mathrm{~b}$ is of $10.8 \%$, thus leading to a desirable analytical $\mathrm{CV}\left(\mathrm{CV}_{\mathrm{A}}\right)$ of $5.4 \%$.

(3) Linearity was evaluated according to the CLSI-6A guideline: two samples, presenting a concentration of 11.30 and $5.08 \mathrm{U} / \mathrm{L}$, respectively were diluted with a saline solution according to this scheme: Pure A $0.9 \mathrm{~A}+0.01 \mathrm{~B}, 0.8 \mathrm{~A}+0.2 \mathrm{~B}, 0.7 \mathrm{~A}+0.3 \mathrm{~B}$, $0.6 \mathrm{~A}+0.4 \mathrm{~B}, 0.5 \mathrm{~A}+0.5 \mathrm{~B}, 0.4 \mathrm{~A}+0.6 \mathrm{~B} ; 0.3 \mathrm{~A}+0.7 \mathrm{~B}, 0.2 \mathrm{~A}+0.8 \mathrm{~B}$, $0.1 \mathrm{~A}+0.9 \mathrm{~B}$ and Pure $\mathrm{B}$.

(4) We used the remnant serum samples from a Belgian population from European lineage to establish the reference ranges in "apparently healthy" men, pre and post-menopause women. These samples were measured in duplicates. Since we did not have the information on the menstrual status of the women, we arbitrarily selected a population of women $<45$ years old and a population of women $>60$ years old to separate the "pre" to "post" menopause status. We excluded from this population any subject presenting CKD, cancer of any origin, osteoporosis or any other disease affecting bone metabolism, rheumatoid arthritis or any other inflammatory disease or who was treated by a drug that could influence the bone metabolism. It was unfortunately impossible to retrospectively define the fasting status of the subjects nor the exact time of sampling.

(5) We compared the Nittobo and the IDS iSYS systems in remnant samples from different clinical populations: 30 hemodialyzed patients (HD), 40 stage 3-5 CKD patients, 40 patients suffering from rheumatoid arthritis (RA), 40 osteoporotic (OP) and 80 postmenopausal women (PM).

(6) Finally, to evaluate the impact of renal function on different bone markers, we measured TRACP-5b, TRACP-5a (Hycult Biotec, Uden, Netherlands), $\beta$-CTX (IDS, iSYS), bone alkaline phosphatase (b-ALP; IDS iSYS) and 3rd generation PTH (DiaSorin Liaison, Saluggia, Italy) in a population of 20 hemodialyzed and 40 CKD patients The results obtained were compared to the reference ranges proposed by the different manufacturers and plotted against the estimated glomerular filtration rate of the patients [21]. 


\section{Stability}

All samples used in this study have been kept for a maximum of 3 months at $-80^{\circ} \mathrm{C}$ before measurement.

\section{Statistics}

We used Medcalc Version 18.6 (Ostende, Belgium) for the statistical calculations. Passing-Bablok regression and Bland-Altman graphs were used to compare the methods.

\section{Ethics}

Remnant samples only were used in this study. No specific approval was requested to the $\mathrm{CHU}$ de Liège Institutional Review Board as a leaflet including the following statement is given to all admitted patients: "According to the law of the December 19, 2008, any left-over of biological material collected from patients for their standard medical management and normally destroyed when all diagnostic analysis have been performed, can be used for validation of methods. The law authorizes such use except if the patient expressed an opposition when still alive (presumed consent). Written informed consent for participation was not required for this study in accordance with the Belgian national legislation and the Institutional requirements".

\section{Results}

The analytical validation of the Nittobo EIA assay provided the following results: a LOD and a LOQ at 0.02 and 0.35 $\mathrm{U} / \mathrm{L}$, respectively and CVs ranging from $8.3 \%$ (at $0.84 \mathrm{U} / \mathrm{L}$ )

Table 1: Precision profile of the Nittobo TRACP-5b assay.

\begin{tabular}{lrrrrr}
\hline Sample & $\begin{array}{r}\text { Mean, } \\
\text { U/L }\end{array}$ & $\begin{array}{r}\text { Intraday } \\
\text { SD, U/L }\end{array}$ & $\begin{array}{r}\text { Interday } \\
\text { SD, U/L }\end{array}$ & $\begin{array}{r}\text { Intraday } \\
\text { CV, \% }\end{array}$ & $\begin{array}{r}\text { Interday } \\
\text { CV, \% }\end{array}$ \\
\hline 1 & 0.841 & 0.0386 & 0.0701 & 4.6 & $\mathbf{8 . 3}$ \\
2 & 2.006 & 0.0994 & 0.1085 & 5.0 & 5.4 \\
3 & 5.64 & 0.2596 & 0.3647 & 4.6 & 6.5 \\
4 & 9.95 & 0.3415 & 0.4296 & 3.4 & 4.3 \\
5 & 15.03 & 0.6211 & 0.6506 & 4.1 & 4.3 \\
\hline
\end{tabular}

The results in bold are higher than the desirable analytical coefficient of variation based on biological variability (5.4\%). to $4.3 \%$ (at $15.0 \mathrm{U} / \mathrm{L}$ ) (Table 1). The samples at 0.84 and 5.64 $\mathrm{U} / \mathrm{L}$ showed a CV higher than $5.4 \%$. Mean recovery of the expected vs. found concentrations were 97.9 and $91.5 \%$ and the method was found to be linear up to a concentration of $11.3 \mathrm{U} / \mathrm{L}$.

The results of the reference interval study are presented in Table 2. The mean CV on the duplicates was $5.3 \pm 1.6 \%$. Distribution of the results was not normal in any of the three sub-groups according to the Kolmogorov-Smirnov test. In men, we did not observe any correlation between age and TRACP-5b values and those older than 51 years old did not present values different from those $<50$ years old.

On the contrary, women older than 60 years old presented significantly higher values than those $<45$ years old ( $3.4 \pm 1.6$ vs. $2.4 \pm 1.1 \mathrm{U} / \mathrm{L}, \mathrm{p}=0.0002)$

The Passing-Bablok regression between IDS iSYS and Nitttobo according to patient type is presented in Figure 1. The regression equation obtained in the 189 samples was Nittobo $=1.13(95 \%$ CI: $1.09-1.16) \times$ IDS iSYS -0.4 (95\% CI: $-0.5 ;-0.3)$. The Bland-Altman plot showed a mean difference (iSYS-Nittobo) of $-0.01 \mathrm{U} / \mathrm{L}$ (95\% of the differences ranging from -0.73 to $0.71 \mathrm{U} / \mathrm{L}$ ) (Figure 2).

The relation between eGFR and TRACP-5b, $\beta$-CTX, b-ALP and PTH in $20 \mathrm{HD}$ and 40 CKD patients is presented in Figure 3. As expected, PTH values tended to increase with decreasing GFR in CKD patients. Most of the HD patients presented a PTH concentration comprised between 2 and 9 times the upper limit of normality, which correspond to the targets provided by the KDIGO [22]. TRACP-5b and $b$-ALP were lower than the respective upper limit of normality of the assays for most of CKD and HD patients. However, contrary to TRACP-5b and b-ALP, $\beta$-CTX increased with decreasing eGFR.

\section{Discussion}

TRACP-5b is an interesting bone resorption marker, which has been recommended and used for a long time in Japan [13]. This biomarker possesses interesting intrinsic

Table 2: Reference intervals of Nittobo TRACP-5b observed in a Western European population of males, females $<45$ years old and females $>60$ years old.

\begin{tabular}{lrrrrrr}
\hline Population & $\begin{array}{r}\text { Number of } \\
\text { subjects }\end{array}$ & $\begin{array}{r}\text { Age range, } \\
\text { years }\end{array}$ & $\begin{array}{r}\text { Age } \\
\text { (mean } \pm \text { SD) }\end{array}$ & $\begin{array}{r}\text { TRACP-5b } \\
\text { Range, U/L }\end{array}$ & $\begin{array}{r}\text { Reference range }(90 \% \text { CI) after } \\
\text { log-transformation, U/L }\end{array}$ & $\begin{array}{r}\text { Upper and lower limits according } \\
\text { to the non parametric percentile } \\
\text { method (CLSI C28-A3), U/L }\end{array}$ \\
\hline $\begin{array}{l}\text { Males } \\
\begin{array}{l}\text { Females }<45 \\
\text { years old }\end{array}\end{array} \quad 119$ & $18.6-90.3$ & $53.4 \pm 17.7$ & $0.7-13.7$ & $0.9(0.7 ; 1.0)-7.1(6.2 ; 8.2)$ & $0.8-7.6$ \\
$\begin{array}{l}\text { Females }>60 \\
\text { years old }\end{array}$ & 50 & $18.7-44.0$ & $33.0 \pm 7.4$ & $0.9-4.8$ & $0.8(0.7 ; 1.0)-5.5(4.5 ; 6.6)$ & $0.9-4.7$ \\
\hline
\end{tabular}




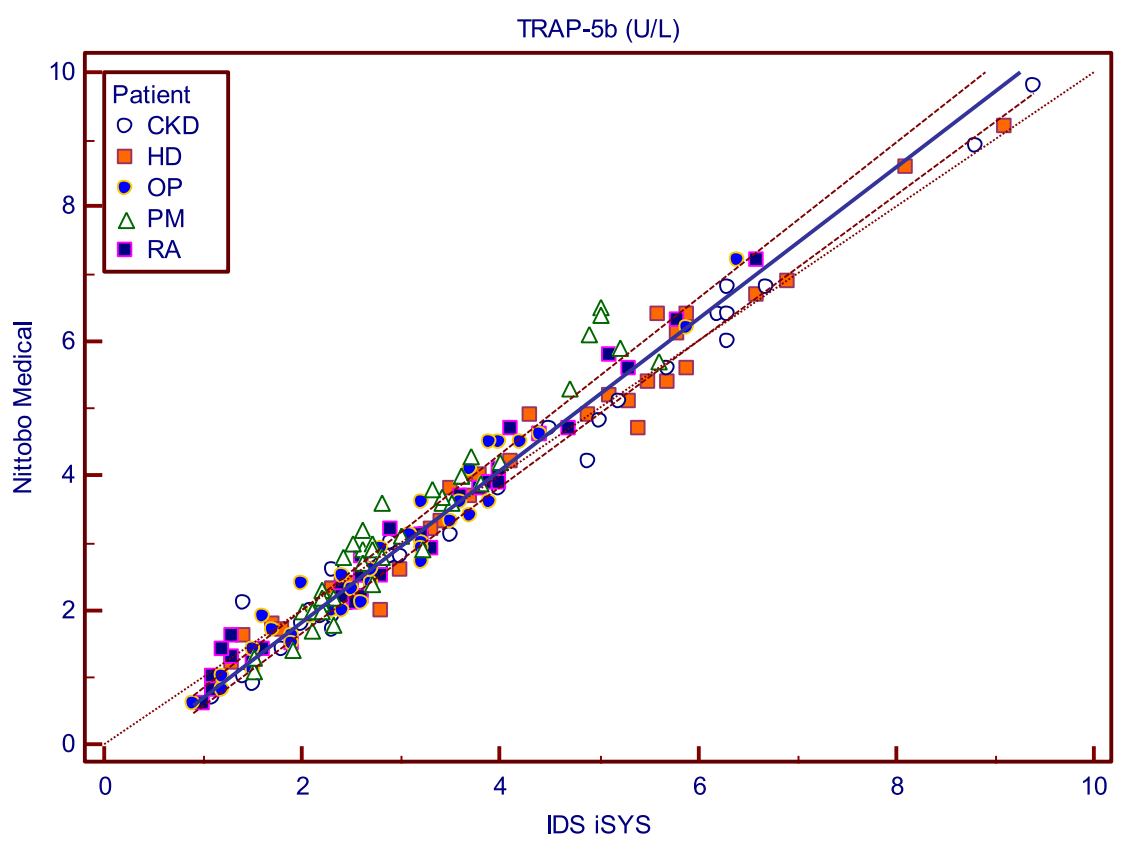

Figure 1: Passing-Bablok regression of TRACP-5b measured by IDS iSYS and Nittobo in different patients.

Patients suffering from chronic kidney diseases (CKD), hemodialyzed patients (HD), patients suffering from osteoporosis (OP), postmenopause women (PM) and patients suffering from rheumatoid arthritis (RA)

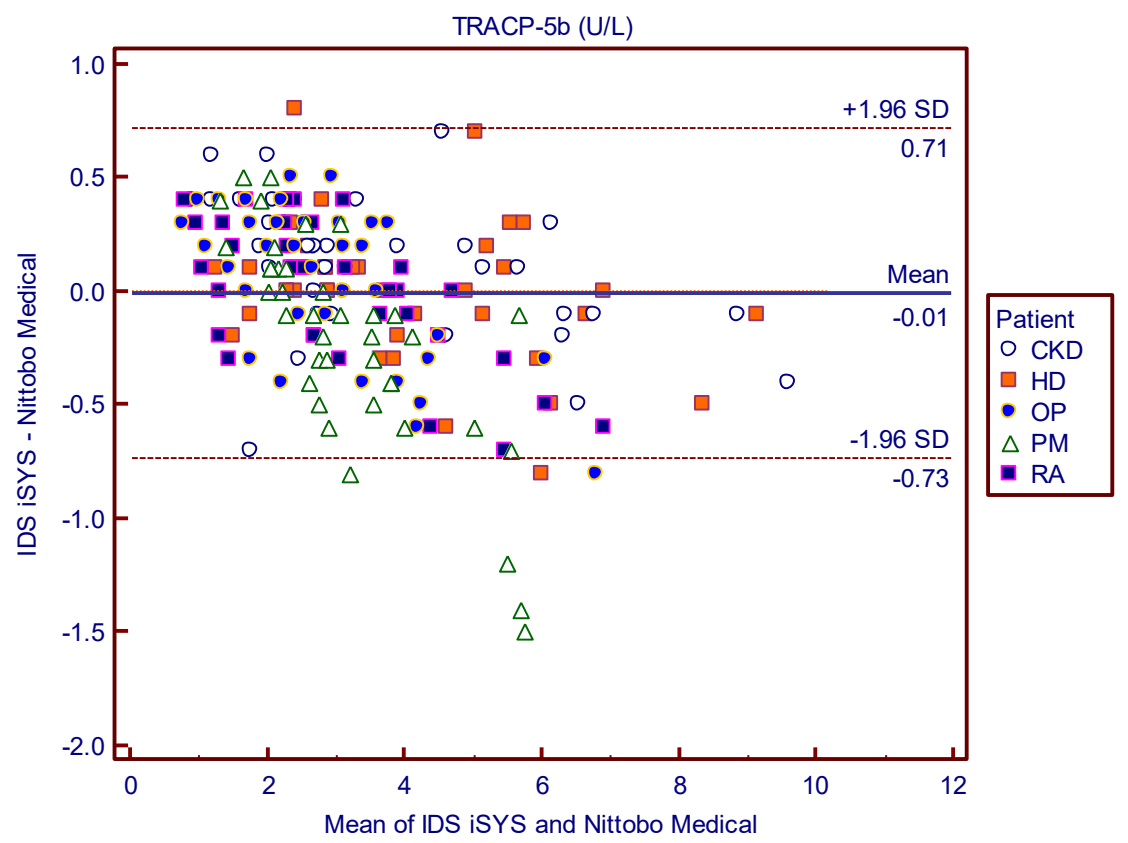

Figure 2: Bland-Altman plot of TRACP-5b measured by IDS iSYS and Nittobo in different patients.

Patients suffering from chronic kidney diseases (CKD), hemodialyzed patients (HD), patients suffering from osteoporosis (OP), postmenopause women (PM) and patients suffering from rheumatoid arthritis (RA).

properties and is more and more evaluated in research and clinical practice, especially for the monitoring of bone turnover in CKD patients [17, 18, 23-26]. Our results show that the Nittobo assay presents interesting analytical features compatible with the clinical practice even if our validation data show that two samples presented a $\mathrm{CV}$ higher than the desirable $\mathrm{CV}$ according to biological variation. 

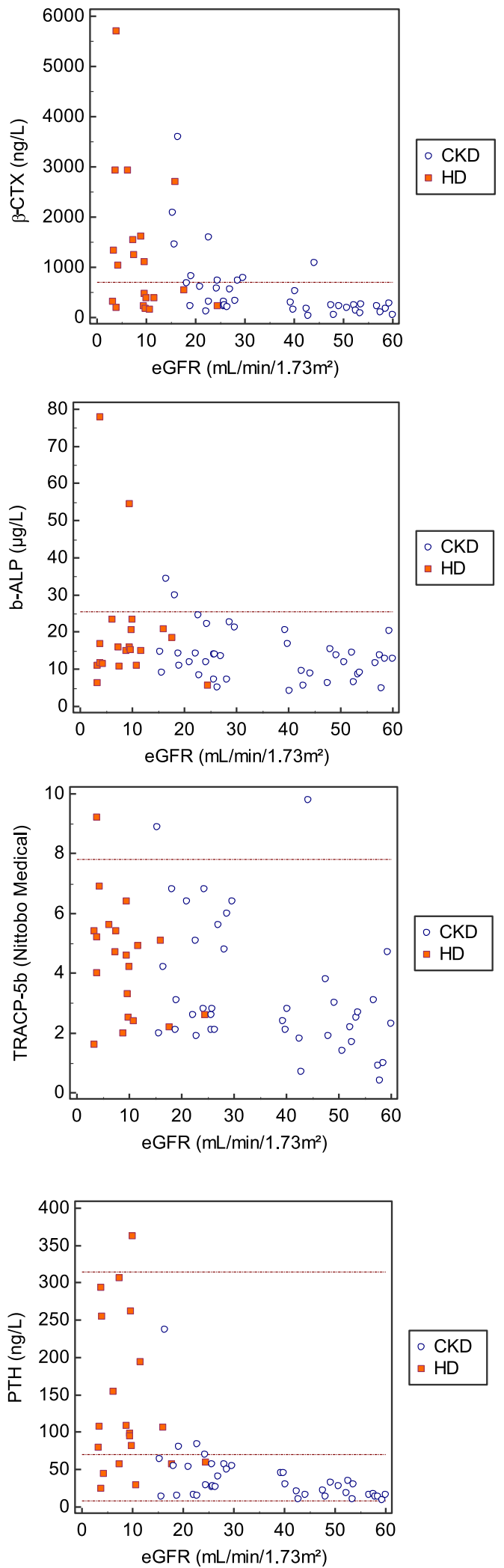

Figure 3: Comparison of the results obtained in a population of CKD and HD patients for different bone markers according to their respective level of eGFR.

The dotted lines represent the upper limit of normality ( $\beta$-CTX, b-ALP and TRAPcp-5b) or 2 and 9 times the upper limit of the assay (PTH).
We have also established reference ranges for a Western-European population of healthy males, pre and post-menopause females. If the number of subjects included in the men and postmenopause women was $\geq 119$, in accordance with the CLSI EP28-A3 Guideline, the number of pre-menopause women was lower and only included 50 individuals. The upper limits of normality we obtained for these specific populations were higher than those observed in a very large cohort of Japanese healthy individuals [27]. Different environmental or ethnical factors could explain these differences. Since TRACP-5b reflects osteoclasts number, the mean larger size of the skeleton of WesternEuropean vs. Japanese individuals could also be an explanation. These results highlight the importance of performing local reference ranges.

Two methods are currently available for TRACP-5b determination, and to the best of our knowledge, these methods had never been compared together. We observed a proportional bias between IDS iSYS and Nittobo Medical results. The rather small confidence interval around the slope and the small intercept clearly indicate that the harmonization of the results obtained by the two methods is possible by using a common commutable calibrator. This would be another interesting feature of TRACP- $5 b$ since harmonization of other bone biomarkers like $\beta$-CTX [28], b-ALP [29] or PINP assays [30] remains difficult.

Finally, our results suggest that TRACP-5b and b-ALP were not affected by renal function since their concentration in patients suffering from chronic kidney diseases and hemodialyzed patients remained in the range expected for healthy individuals. This was however not the case for $\beta$-CTX since most of the patients with a GFR lower than an approximate threshold of $30 \mathrm{~mL} / \mathrm{min} / 1.73 \mathrm{~m}^{2}$ and most of hemodialyzed patients presented values higher than the 95th percentile of healthy post-menopause women.

In conclusion, our data show that the Nittobo TRACP-5b EIA presents interesting analytical characteristics. The comparison with the IDS iSYS assay in different populations suggest that the results provided by the two methods could be harmonized. Contrary to $\beta$-CTX TRACP- $5 \mathrm{~b}$ does not seem to be affected by kidney function and we provided, for the first time, robust reference intervals for the Nittobo Medical assay in a Western-European population.

Acknowledgments: We thank Nittobo Medical for providing the reagents.

Research funding: None declared.

Author contributions: EC designed the study, supervised it and wrote the paper. PL performed the analytical determinations. PD critically reviewed the manuscript. All 
authors have accepted responsibility for the entire content of this manuscript and approved its submission.

Competing interests: EC is consultant for DiaSorin, IDS, Fujirebio, Nittobo. PD is consultant for IDS.

Informed consent: Not applicable.

Ethical approval: Remnant samples only were used in this study. No specific approval was requested to the CHU de Liège Institutional Review Board as a leaflet including the following statement is given to all admitted patients: "According to the law of the December 19, 2008, any leftover of biological material collected from patients for their standard medical management and normally destroyed when all diagnostic analysis have been performed, can be used for validation of methods. The law authorizes such use except if the patient expressed an opposition when still alive (presumed consent). Written informed consent for participation was not required for this study in accordance with the Belgian national legislation and the Institutional requirements".

\section{References}

1. Cassady Al, King AG, Cross NCP, Hume DA. Isolation and characterization of the genes encoding mouse and human type-5 acid phosphatase. Gene 1993;130:201-7.

2. Janckila AJ, Yam LT. Biology and clinical significance of tartrateresistant acid phosphatases: new perspectives on an old enzyme. Calcif Tissue Int 2009;85:465-83.

3. Yaziji H, Janckila AJ, Lear SC, Martin AW, Yam LT. Immunohistochemical detection of tartrate-resistant acid phosphatase in non-hematopoietic human tissues. Am J Clin Pathol 1995;104:397-402.

4. Lam WKW, Eastlund DT, Li C, Yam LT. Biochemical propertiesof tartrate-resistant acid phosphatasein serum of adults and children. Clin Chem 1978;24:1105-8.

5. Chu P, Chao TY, Lin YF, Janckila AJ, Yam LT. Correlation between histomorphometric parameters of bone resorption and serum type $5 \mathrm{~b}$ tartrate-resistant acid phosphatase in uremic patients on maintenance hemodialysis. Am J Kidney Dis 2003;41:1052-9.

6. Halleen JM, Ylipahkala H, Alatalo SL, Janckila AJ, Heikkinen JE, Suominen $\mathrm{H}$, et al. Serum tartrate-resistant acid phosphatase $5 \mathrm{~b}$, but not $5 \mathrm{a}$, correlates with other markers of bone turnover and bone mineral density. Calcif Tissue Int 2002;71:20-5.

7. Saunders PTK, Renegar RH, Raub TJ, Baumbach GA, Atkinson PH, Bazer FW, et al. The carbohydrate structure of porcine uteroferrin and the role of the high mannose chains in promoting uptake by the reticuloendothelial cells of the fetal liver. J Biol Chem 1985; 260:3658-65.

8. Halleen J, Hentunen TA, Hellman J, Väänänen HK. Tartrate-resistant acid phosphatase from human bone: purification and development of an immunoassay. J Bone Miner Res 1996;11:1444-52.

9. Vasikaran $S$, Eastell R, Bruyère 0 , Foldes a. J, Garnero $P$, Griesmacher A, et al. Markers of bone turnover for the prediction of fracture risk and monitoring of osteoporosis treatment: a need for international reference standards. Osteoporos Int 2011;22: 391-420.

10. Hannon RA, Clowes JA, Eagleton AC, Al Hadari A, Eastell R, Blumsohn A. Clinical performance of immunoreactive tartrateresistant acid phosphatase isoform $5 \mathrm{~b}$ as a marker of bone resorption. Bone 2004;34:187-94.

11. Cavalier E, Delanaye P, Moranne 0 . Variability of new bone mineral metabolism markers in patients treated with maintenance hemodialysis: implications for clinical decision making. Am J Kidney Dis 2013;61:847-8.

12. Halleen JM, Alatalo SL, Suominen H, Cheng S, Janckila AJ, Väänänen KH. Tartrate-resistant acid phosphatase as a serum marker of bone resorption. J Bone Miner Res 2000;15:1337-45.

13. Nishizawa Y, Miura M, Ichimura S, Inaba M, Imanishi Y, Shiraki M, et al. Executive summary of the Japan Osteoporosis Society guide for the use of bone turnover markers in the diagnosis and treatment of osteoporosis (2018 edition). Clin Chim Acta 2019; 498:101-7.

14. Shidara K, Inaba M, Okuno S, Yamada S, Kumeda Y, Imanishi Y, et al. Serum levels of TRAP5b, a new bone resorption marker unaffected by renal dysfunction, as a useful marker of cortical bone loss in hemodialysis patients. Calcif Tissue Int 2008;82: 278-87.

15. Evenepoel P, Claes K, Meijers B, Laurent MR, Bammens B, Naesens $M$, et al. Natural history of mineral metabolism, bone turnover and bone mineral density in de novo renal transplant recipients treated with a steroid minimization immunosuppressive protocol. Nephrol Dial Transplant 2020;35:697-705.

16. Morris HA, Eastell R, Jorgesen NR, Cavalier E, Vasikaran S, Chubb SAP, et al. Clinical usefulness of bone turnover marker concentrations in osteoporosis. Clin Chim Acta 2017;467:34-41.

17. Salam S, Gallagher O, Gossiel F, Paggiosi M, Khwaja A, Eastell R. Diagnostic accuracy of biomarkers and imaging for bone turnover in renal osteodystrophy. J Am Soc Nephrol 2018;29:1557-65.

18. Jørgensen HS, Behets G, Viaene L, Bammens B, Claes K, Meijers $\mathrm{B}$, et al. Diagnostic accuracy of noninvasive bone turnover markers in renal osteodystrophy. Am J Kidney Dis 2021. https:// doi.org/10.1053/j.ajkd.2021.07.027.

19. Nishizawa Y, Inaba M, Ishii M, Yamashita H, Miki T, Goto H, et al. Reference intervals of serum tartrate-resistant acid phosphatase type $5 \mathrm{~b}$ activity measured with a novel assay in Japanese subjects. J Bone Miner Metab 2008;26:265-70.

20. Fraser CG, Petersen H, Libeer J-C, Ricosl C. Proposals for setting generally applicable quality goals solely based on biology. Pers View Ann Clin Biochem 1997;34:8-12.

21. Cavalier E, Lukas P, Carlisi A, Gadisseur R, Delanaye P. Aminoterminal propeptide of type I procollagen (PINP) in chronic kidney disease patients: the assay matters. Clin Chim Acta 2013; 425:117-8.

22. KDIGO 2017 clinical practice guideline update for the diagnosis, evaluation, prevention, and treatment of chronic kidney diseasemineral and bone disorder (CKD-MBD). Kidney Int Suppl. 2017;7: 1-59.

23. Evenepoel P, Cavalier E, D’Haese PC. Biomarkers predicting bone turnover in the setting of CKD. Curr Osteoporos Rep. Current Osteoporosis Reports 2017;15:178-86.

24. Evenepoel P, Cavalier E, D'Haese PC. Bone biomarkers in de novo renal transplant recipients. Clin Chim Acta 2019;501:179-85. 
25. Evenepoel P, Claes K, Meijers B, Laurent MR, Bammens B, Naesens $M$, et al. Bone mineral density, bone turnover markers, and incident fractures in de novo kidney transplant recipients. Kidney Int 2019;95:1461-70.

26. Khalil R, Antonio L, Laurent MR, David K, Kim NR, Evenepoel P, et al. Early effects of androgen deprivation on bone and mineral homeostasis in adult men: a prospective cohort study. Eur J Endocrinol 2020;183:181-9.

27. Kikuchi W, Ichihara K, Mori K, Shimizu Y. Biological sources of variations of tartrate-resistant acid phosphatase $5 \mathrm{~b}$ in a healthy Japanese population. Ann Clin Biochem 2021;58:358-67.

28. Cavalier E, Eastell R, Jørgensen NR, Makris K, Tournis S, Vasikaran S, et al. A multicenter study to evaluate harmonization of assays for
C-terminal telopeptides of type I collagen (B-CTX): a report from the IFCC-IOF committee for bone metabolism (C-BM). Calcif Tissue Int 2021;108:785-97.

29. Cavalier E, Souberbielle J-CC, Gadisseur R, Dubois B, Krzesinski J-MM, Delanaye P. Inter-method variability in bone alkaline phosphatase measurement: clinical impact on the management of dialysis patients. Clin Biochem 2014;47: 1227-30.

30. Cavalier E, Eastell R, Jørgensen NR, Makris K, Tournis S, Vasikaran $\mathrm{S}$, et al. A multicenter study to evaluate harmonization of assays for $\mathrm{N}$-terminal propeptide of type I procollagen ( P1NP): a report from the IFCC-IOF Joint Committee for Bone Metabolism. Clin Chem Lab Med 2019;25:1546-55. 